\title{
Observations and Numerical Models of Solar Coronal Heating Associated with Spicules
}

\author{
B. De Pontieu ${ }^{1,2}$ (1) , I. De Moortel $^{3}$ (10, J. Martinez-Sykora ${ }^{1,4}$ (1) , and S. W. McIntosh ${ }^{5}$ (1) \\ ${ }^{1}$ Lockheed Martin Solar \& Astrophysics Laboratory, 3251 Hanover Street, Org. A021S, Building 252, Palo Alto, CA 94304, USA \\ ${ }^{2}$ Institute of Theoretical Astrophysics, University of Oslo, Blindern, P.O. Box 1029 Blindern, NO-0315 Oslo, Norway \\ ${ }^{3}$ School of Mathematics and Statistics, University of St Andrews, St Andrews, Fife KY16 9SS, UK \\ ${ }^{4}$ Bay Area Environmental Research Institute, Sonoma, CA 94952, USA \\ ${ }^{5}$ High Altitude Observatory, National Center for Atmospheric Research, P.O. Box 3000, Boulder, CO 80307, USA \\ Received 2017 June 5; revised 2017 July 13; accepted 2017 July 13; published 2017 August 18
}

\begin{abstract}
Spicules have been proposed as significant contributors to the mass and energy balance of the corona. While previous observations have provided a glimpse of short-lived transient brightenings in the corona that are associated with spicules, these observations have been contested and are the subject of a vigorous debate both on the modeling and the observational side. Therefore, it remains unclear whether plasma is heated to coronal temperatures in association with spicules. We use high-resolution observations of the chromosphere and transition region (TR) with the Interface Region Imaging Spectrograph and of the corona with the Atmospheric Imaging Assembly on board the Solar Dynamics Observatory to show evidence of the formation of coronal structures associated with spicular mass ejections and heating of plasma to TR and coronal temperatures. Our observations suggest that a significant fraction of the highly dynamic loop fan environment associated with plage regions may be the result of the formation of such new coronal strands, a process that previously had been interpreted as the propagation of transient propagating coronal disturbances. Our observations are supported by 2.5D radiative MHD simulations that show heating to coronal temperatures in association with spicules. Our results suggest that heating and strong flows play an important role in maintaining the substructure of loop fans, in addition to the waves that permeate this low coronal environment.
\end{abstract}

Key words: Sun: chromosphere - Sun: corona - Sun: magnetic fields - Sun: transition region

Supporting material: animations

\section{Introduction}

Chromospheric spicules are dynamic jet-like features that dominate the solar limb and appear to penetrate the milliondegree corona before falling back to the surface. Their nature has remained a mystery with many explanations proposed for their origin (for reviews, see Sterling 2000; Tsiropoula et al. 2012). They have long been considered a plausible mechanism for providing plasma to the corona (Beckers 1968; Pneuman \& Kopp 1978; Athay \& Holzer 1982). The discussion about their role in the outer atmosphere was recently revived, with the advent of Hinode (Kosugi et al. 2007), in particular the Solar Optical Telescope (SOT; Tsuneta et al. 2008) and the EUV Imaging Spectrometer (EIS; Culhane et al. 2007), the Atmospheric Imaging Assembly (AIA) on board Solar Dynamics Observatory (Lemen et al. 2012), and the Interface Region Imaging Spectrograph (IRIS; De Pontieu et al. 2014), as well as advanced 3D radiative MHD simulations (e.g., Martínez-Sykora et al. 2011, 2012, 2013).

Observations with these spacecraft have provided a new view on spicules, revealing the presence of (1) relatively slow $\left(<40 \mathrm{~km} \mathrm{~s}^{-1}\right)$, longer-lived (5-10 minute) spicules that do not appear to show significant heating to transition region (TR) or coronal temperatures, falling back toward the surface as chromospheric features (type I spicules, or dynamic fibrils when seen on the disk; Hansteen et al. 2006); (2) fast (40-100 $\mathrm{km} \mathrm{s}^{-1}$; type II) spicules that are only briefly visible (1-2 minutes) in chromospheric observables such as $\mathrm{Ca}$ II $\mathrm{H}$ $3968 \AA$ (De Pontieu et al. 2007; Pereira et al. 2012) and in which a fraction of the plasma appears to be heated to at least TR temperatures (Pereira et al. 2014; Rouppe van der Voort et al. 2015; Skogsrud et al. 2015) before returning to the surface. We focus here on the impact of type II spicules on the coronal mass and energy balance.

There have been several suggestions that these spicules play a significant role in heating the corona. De Pontieu et al. (2009) suggested that strong upflows of (multi)million-degree plasma seen at the footpoints of coronal loops as spectral line asymmetries (Hara et al. 2008) were associated with upper chromospheric activity (e.g., McIntosh \& De Pontieu 2009). De Pontieu et al. (2011) suggested that the disk counterparts of spicules (rapid blueshifted events or RBEs, see Rouppe van der Voort et al. 2009) are associated with brightenings in coronal SDO/AIA lines in active regions (for quiet Sun, see Henriques et al. 2016). These studies have been the subject of significant debate, both from an observational and a theoretical point of view. Madjarska et al. (2011) studied coronal hole spicules at the limb and found no evidence for coronal counterparts using lower resolution and lower signal-to-noise observations from $\mathrm{SOHO} / \mathrm{SUMER}$ (Wilhelm et al. 1995). Klimchuk (2012) used simplified theoretical considerations to reject a significant role of spicules in the coronal heating issue, while Tripathi \& Klimchuk (2013) and Patsourakos et al. (2014) studied spectral line asymmetries from EIS and argued that while spicules may play a role in the coronal mass and energy balance, it is likely not a dominant one. Lacking a theoretical model that captures the complexity of the spicular environment, these studies are based on simplifying assumptions about the physical scenario focusing on single-field-line approaches that underestimate the complexity of the spicular environment. The latter is observationally challenging to capture: they rapidly fade in and out of various passbands, necessitating a multi-instrument approach, and are so dynamic and finely structured that many instruments do not resolve their spatio-temporal evolution. 


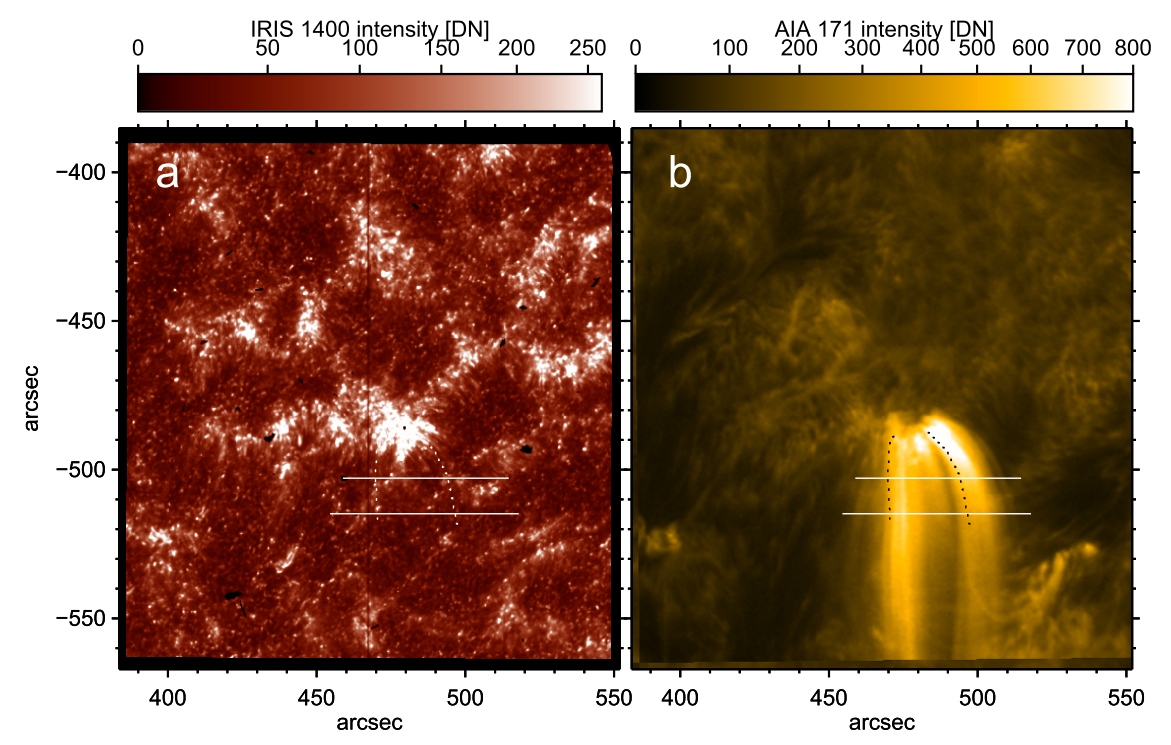

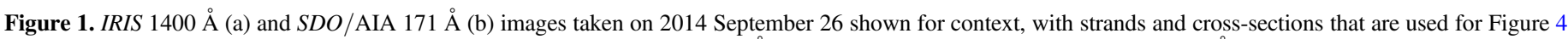
overplotted. Accompanied by an online animation that also shows IRIS $1330 \AA$ and the running difference of SDO/AIA $171 \AA$.

(An animation of this figure is available.)

Here we attempt to address both issues. We exploit the IRIS discovery of the TR counterparts of spicules, in particular the fact that these are longer-lived than the chromospheric spicules, allowing us to more easily track their evolution. We use SDO/ AIA to focus on the impact of spicules on coronal loops that are connected to plage or enhanced network regions. These loops have long been known to be permeated by propagating coronal disturbances (PCDs): rapid $\left(\sim 100 \mathrm{~km} \mathrm{~s}^{-1}\right)$ intensity disturbances whose exact nature remains unknown. While it is clear that PCDs that originate from sunspot umbrae are caused by sound waves, it is less clear whether waves or flows cause these PCDs in plage regions, with both receiving observational and theoretical support (e.g., de Moortel et al. 2002a, 2002b; De Pontieu et al. 2005; De Moortel 2009; De Pontieu \& McIntosh 2010; Verwichte et al. 2010; De Moortel \& Nakariakov 2012; Ofman et al. 2012; Tian et al. 2012; Wang et al. 2013; Petralia et al. 2014; De Moortel et al. 2015; Samanta et al. 2015; Bryans et al. 2016). We also take advantage of recent developments in spicule modeling (Martínez-Sykora et al. 2016, 2017) that appear to show coronal heating associated with spicules.

\section{Observations}

We use IRIS slit-jaw observations of AR 12171 at $x_{\text {cen }}=464^{\prime \prime}, y_{\text {cen }}=-476^{\prime \prime}$, taken on 2014 September 26 from 00:34-01:37 UTC using OBS-ID 3820107266. The IRIS level 2 data were corrected for dark current, flat-field geometry and coaligned as described in De Pontieu et al. (2014). To boost signal to noise, the IRIS data were summed on board $2 \times 2$, so that spatial pixels are 0 !" $33 \times 0$ !" 33 . Both 1330 and $1400 \AA$ passbands were used, which are dominated by far-ultraviolet continuum (formed in the low chromosphere), and C II 1335/ $1336 \AA$ lines (formed at upper chromospheric and low TR temperatures, from 15,000 to 40,000 K; Rathore et al. 2015) and Si IV 1394/1403 $\AA$ lines (formed at TR temperatures from 20,000 to $300,000 \mathrm{~K}$; Olluri et al. 2015), respectively. Co-temporal SDO/AIA observations in the 1600, 171, and $193 \AA$ A passbands were prepped, coaligned, and normalized using the SolarSoft aia_prep routine. The AIA data were interpolated in time and space to match the IRIS/SJI temporal cadence (10.4 s) and spatial resolution (0."33).

We focus on a decayed plage region that is associated with "plume"-like coronal structures that emanate toward the south (Figure 1).

\section{Results}

\subsection{Observations}

Animations of the IRIS $1400 \AA$ passband show that the footpoints of these coronal loops are dominated by a multitude of spicule-like features, which, given their appearance, are most likely caused by $\mathrm{TR}$ emission $\left(\mathrm{Si}^{3+}\right.$ ions). These spicules originate from magnetic flux concentrations, shooting away from the weak plage at apparent speeds of 50-200 $\mathrm{km} \mathrm{s}^{-1}$. One can often see the spicules retract after reaching a maximum extent, although not always. They are often not clearly visible along their whole length and fade as they extend away from the plage, suggesting a complex thermal environment and evolution, involving heating and/or cooling. The coronal loops rooted in the same region similarly show a lot of complexity, which traditionally have been associated with "propagating coronal disturbances" along pre-existing coronal loops. However, closer inspection of the time series associated with Figure 1 shows that much of this activity is actually caused by a variety of coronal strands appearing and disappearing, typically starting from the bottom of the loops, followed by "propagation" away from the footpoint. It is also clear that the PCDs that are observed here are not as cleanly periodic as those associated with sunspot umbrae (see also De Pontieu \& McIntosh 2010). This is illustrated in Figures 2 and 3 and accompanying animations.

The line-of-sight superposition and the multitude of events overlapping in space and time means it is not straightforward to disentangle individual events. However, analysis of the evolution of several of the larger spicules that stand out individually show an intriguing connection between the $\mathrm{C}$ II and Si IV spicules in IRIS slit-jaw animations and the formation of strands in the 

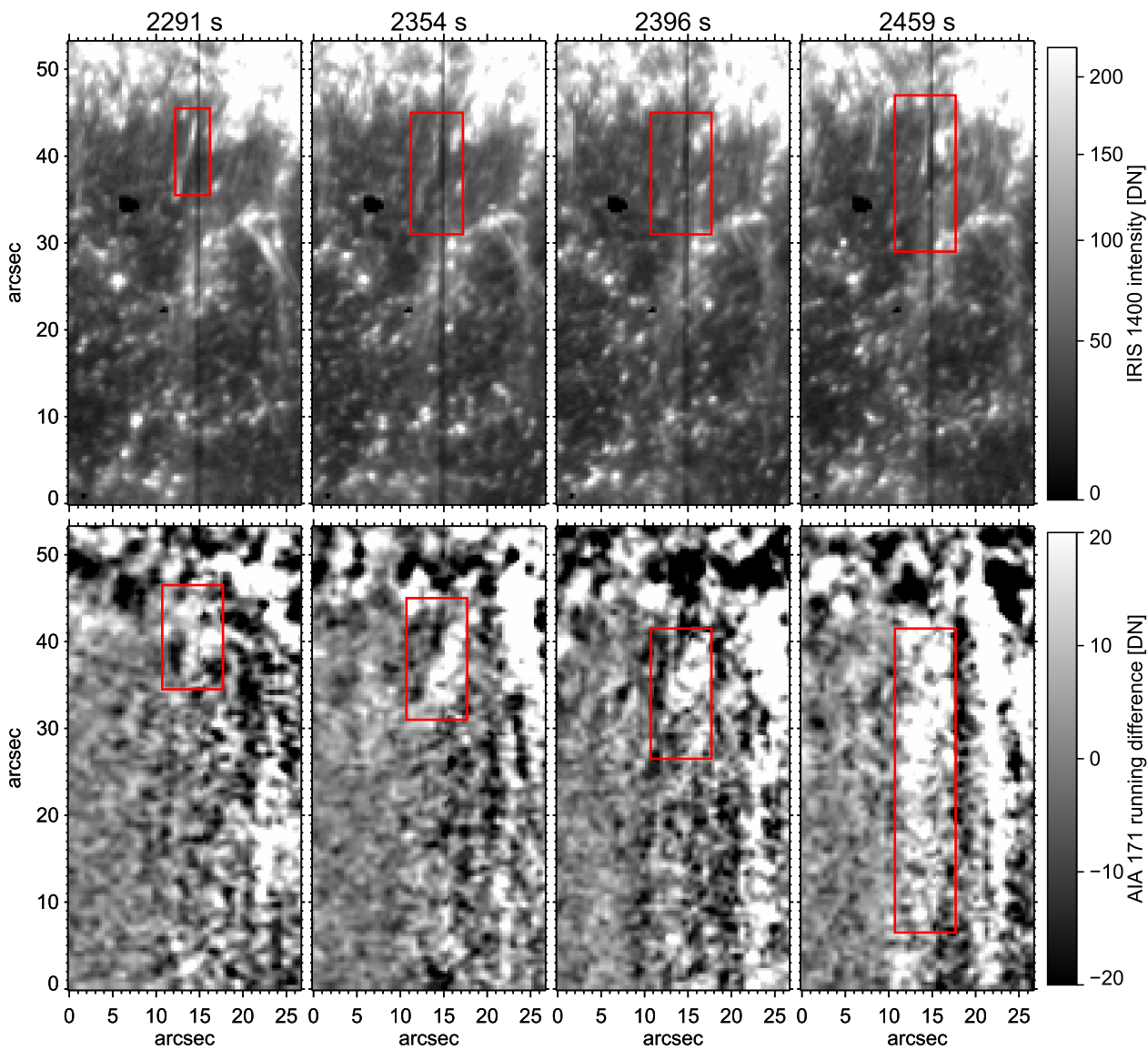

Figure 2. Top: IRIS $1400 \AA$ images with the red boxes indicating the formation of the TR counterpart of a type II spicule. Bottom: SDO/AIA $171 \AA$ A runningdifference images with red boxes indicating the formation and propagating of a PCD. This figure is accompanied by two animations that show the evolution of this event and a second, unrelated event.

(Animations ( $\mathrm{a}$ and $\mathrm{b})$ of this figure are available.)

coronal loop system (AIA). This is illustrated in Figure 2 and the accompanying animations, which show the temporal evolution of the event shown in Figure 2 as well as a second event. In both cases, we show the Si IV images (top) and the running difference of the $171 \AA$ AIA channel—calculated by differencing the current image with that taken $62 \mathrm{~s}$ earlier, a commonly used method to enhance the visibility of the intensity disturbances. For both cases, we see the spicule form with apparent velocities in the plane-of-the-sky of $\sim 50 \mathrm{~km} \mathrm{~s}^{-1}$, accompanied by a brightening in $171 \AA$ that initially grows with the same apparent speed as the spicule. Toward the time of maximum extension of the spicule $(t=2396 \mathrm{~s})$, the spicule seems to stay roughly constant in length, and we see (red rectangles in Figure 2 and the animations) that the coronal counterpart grows rapidly to cover $30^{\prime \prime}$ in $50 \mathrm{~s}$, suggesting an apparent speed of the order of $400 \mathrm{~km} \mathrm{~s}^{-1}$. A similar evolution can be seen in the second animation accompanying Figure 2: that spicule grows with an apparent speed of the order of $40 \mathrm{~km} \mathrm{~s}^{-1}$ until $t \sim 1980 \mathrm{~s}$ and appears to stay at its maximum extent for a while, and then the coronal counterpart grows another $30^{\prime \prime}$ in $\sim 40 \mathrm{~s}$, suggesting an apparent speed for this phase of $\sim 500 \mathrm{~km} \mathrm{~s}^{-1}$.

Figure 3 shows how the coronal loop strand that is associated with the spicule of Figure 2 becomes visible in both the AIA $171 \AA$ and $193 \AA$ passbands when using a different color table that accentuates small intensity differences. The loop strand is more clearly visible in $171 \AA$ than in $193 \AA$ suggesting that it reaches temperatures closer to the formation temperature of Fe IX $(\log T=5.9)$ rather than that of Fe XII $(\log T=6.2)$. This figure does not show the running difference, but rather the original AIA intensity. It illustrates how this particular feature is not a disturbance on top of a pre-existing coronal loop structure, but the formation of a completely new coronal strand. Detailed inspection of the AIA time series shows that the formation of such strands is not a rare event, but a common occurrence throughout this loop fan structure.

However, the formation of such a strand can also, deceptively, look like a PCD as illustrated in Figure 4. The example in the top row shows the event that was highlighted in Figures 2 and 3. As shown in Figure 3, this event involved the formation of a new loop strand by $t=2200 \mathrm{~s}$, which briefly appears as a PCD in both the 171 and $193 \AA$ channels. Because of the nature of the running difference, the longevity of the strand is not clear at all from panels (A) and (B). This becomes much clearer in panels (C) and (D), which do not show the running difference and instead of the original 171 and $193 \AA$ intensity, the intensity after unsharp masking to enhance the small spatial scales of the coronal loop strands. We show here the spatio-temporal evolution for a cut (see the upper horizontal line in Figure 1) across the loop strand that we highlighted earlier. We see that this particular strand forms when the PCD hits this location. While the PCD continues to propagate away from this location, and the running-difference plot suggests that a "wave" (perturbation) just passed through a background 


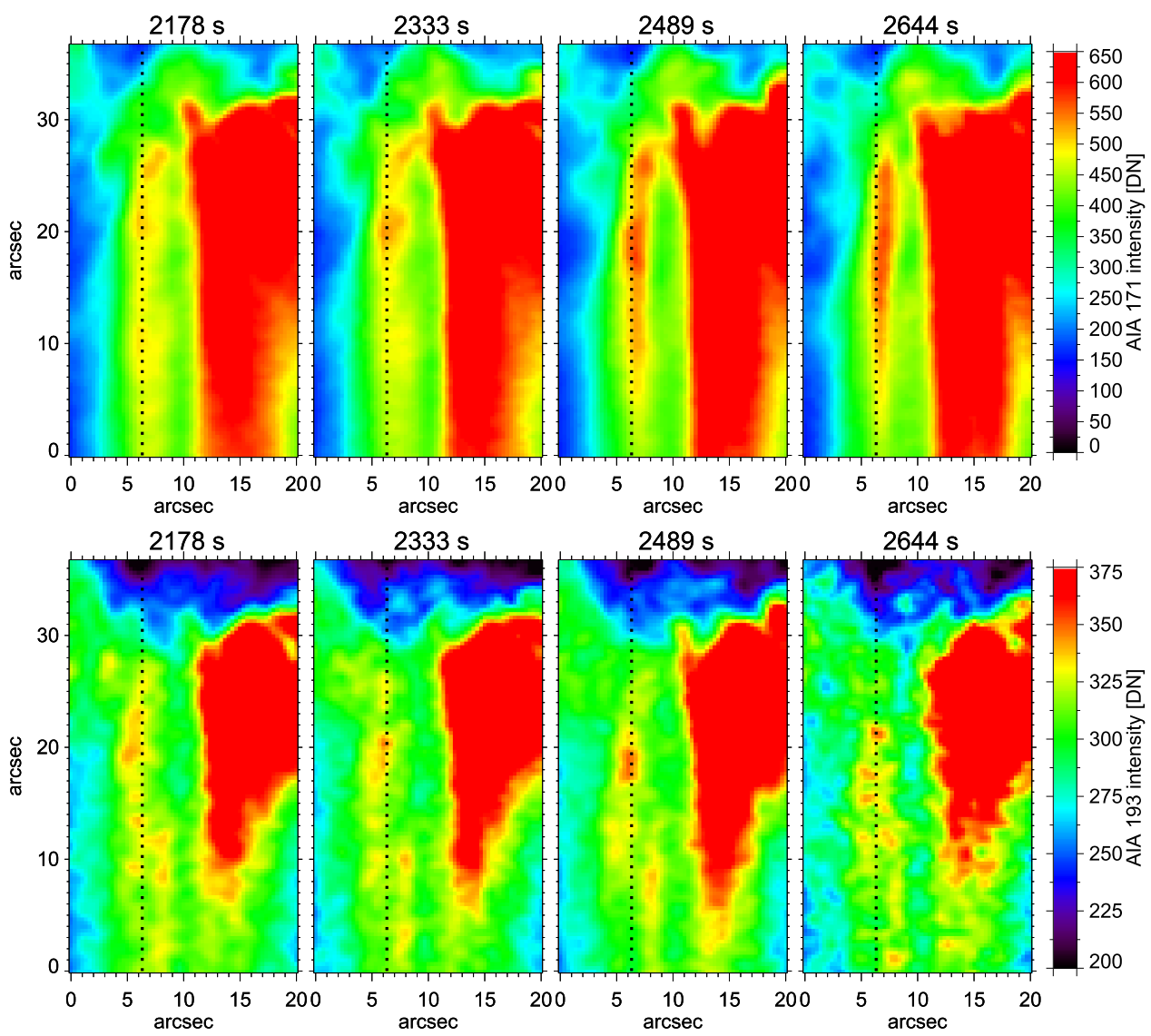

Figure 3. Formation of a coronal loop strand in (top) SDO/AIA $171 \AA$ and (bottom) $193 \AA$. The strand forms underneath and along the dashed vertical line, which is used for the spacetime plot shown at the top of Figure 4.

(An animation of this figure is available.)

structure, panels (C) and (D) indicate that the strand continues to exist long after the PCD has left this region. This particular strand in fact undergoes repeated activity with many PCDs passing through, and the strand being strengthened every time such a PCD passes by.

A more isolated case of strand formation associated with the passage of a PCD is shown at the bottom of Figure 4, which reveals a short-lived passage of a PCD (at $t=1700 \mathrm{~s}$ ) that leads to the formation of a strand, initially brightest in $211 \AA$ (not shown), followed by a brief event in $193 \AA$ (panel $(\mathrm{H})$ ) and then a prolonged presence in $171 \AA$. This event occurs where the right-hand track and bottom horizontal line cross in Figure 1. This sequence of events strongly supports a scenario in which the PCD appears to be associated not only with a spicule, but also with heating of plasma to $\sim 1.5 \mathrm{MK}$ followed by apparently relatively rapid cooling and subsequent fading from the 171 A passband after 10-15 minutes. Many more examples can be found in the data that support this scenario of several phenomena associated with spicules: triggering of PCDs, heating to coronal temperatures, and the formation of loop strands.

\subsection{Simulations}

These results fit well with synthetic Fe IX $171 \AA$ A and Fe XII $193 \AA$ observations from a $2.5 \mathrm{D}$ radiative MHD simulation using Bifrost (Gudiksen et al. 2011), which captures many physical processes important for the dynamics and energetics of the solar atmosphere. This simulation covers a domain from the top of the convection zone into the corona, including selfconsistent chromospheric and coronal heating (MartínezSykora et al. 2016, 2017). We included the effects of interactions between ions and neutrals, or ambipolar diffusion. Ambipolar diffusion plays a key role in the formation of features that closely resemble type II spicules, through a complex mechanism outlined in Martínez-Sykora et al. (2017). In summary, the interaction between weak, granular-scale fields and strong flux concentrations leads to strong magnetic tension, which can emerge into the chromosphere through ambipolar diffusion and leads to a violent release of tension when the low plasma $\beta$ regime is reached in the middle to upper chromosphere. This violent release leads to strong upward acceleration of plasma, the formation of fast spicules, and the generation of strong transverse waves. In addition, currents, created through several mechanisms including wave-mode coupling, gradients in ambipolar diffusion, and the interaction between emerging flux and pre-existing ambient field, are in part dissipated by ambipolar diffusion in the spicule (leading to heating to TR temperatures), and in part propagated into the corona at Alfvén speeds where they lead to significant heating.

The coronal impact of this scenario is illustrated in Figure 5, which includes a running difference of Fe IX and Fe XII intensities along a track that covers a spicule. While the Fe IX response is relatively simple with the apparent propagation of a "PCD" at speeds of $\sim 150 \mathrm{~km} \mathrm{~s}^{-1}$, the Fe XII running difference shows two PCDs, one that is similar in slope to the Fe IX, and another at much higher speeds $\left(\sim 1600 \mathrm{~km} \mathrm{~s}^{-1}\right)$. Comparison 


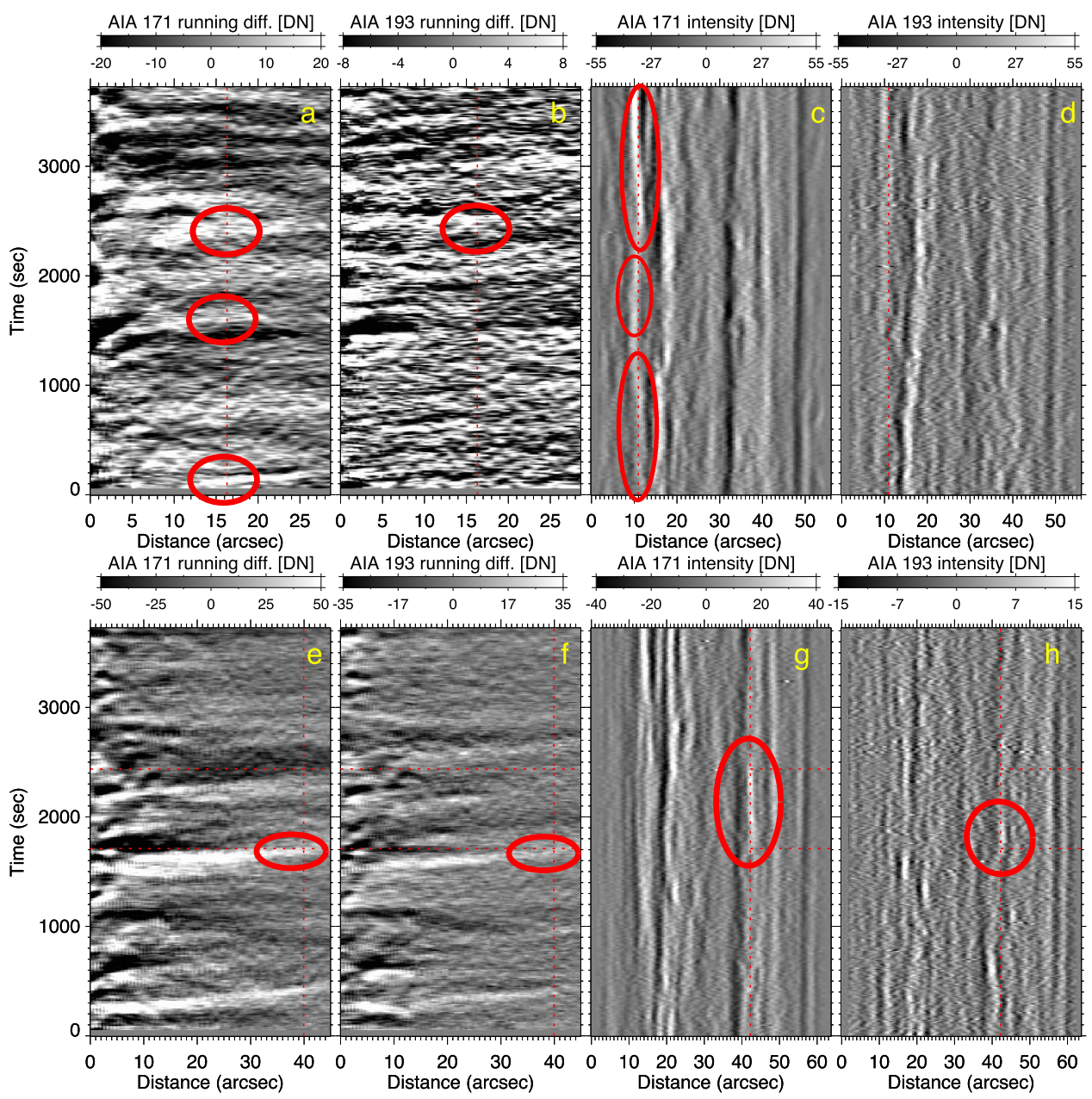

Figure 4. Two examples (top and bottom) of strand formation associated with spicules. (A) Running difference of AIA $171 \AA$ along the left vertical track in Figure 1 and the vertical line in Figure 3, (B) same as for $193 \AA$, (C) cross-section of the unsharp masked AIA $171 \AA$ intensity along the upper horizontal line in Figure 1. (D) Same as for $193 \AA$ A. Panels (E)-(H) show the same but for a different event, highlighted by the right vertical track and the bottom horizontal line in Figure 1. Vertical lines in panels (A), (B), (E), and (F) indicate where the track crosses the horizontal cuts indicated in Figure 1. Horizontal lines and red circles identify specific events described in the text.

with the original intensity of both lines and various physical variables paints a complicated picture and indicates that the interpretation of PCDs in terms of physical mechanisms is not straightforward, as explained below. Both PCDs are causally linked to the launch of a fast spicule. The chromospheric part of this spicule is visible as a parabolic path in the temperature (e) that starts at the same time and location as the Fe IX PCD. The cause of the Fe IX PCD is, at low heights, a mix of flows associated with the TR and coronal counterparts of the spicule (visible as a parabolic path that reaches distances of $10 \mathrm{Mm}$ in the original intensity of Fe IX (a) and in the density (f)), and the coronal remnant of the shock wave that was involved in the spicule acceleration. These flows are caused by the acceleration, compression, and heating of plasma associated with the spicule eruption. At greater heights $(>10 \mathrm{Mm})$, the Fe IX PCD is mostly determined by the shock-wave-related disturbance (similar to De Pontieu et al. 2005; Petralia et al. 2014) as the coronal counterpart of the spicule fades beyond distances of $12 \mathrm{Mm}$. The Fe XII PCDs are even more complex: the slower Fe XII PCD is a mix of spicular flows and shock-wave related disturbances. However, the average slope that is drawn through the slow PCD in this wavelength ignores the fact that the different physical mechanisms lead to different slopes in the spacetime plots, with the apparent speed increasing with distance from the spicule footpoints. The Fe XII running difference (b) also reveals a faster "PCD" that is caused by the rapid formation of a loop strand. This loop strand is formed because of the heating associated with the arrival and dissipation in the corona of the current that also heats the spicular plasma, as well as thermal conduction that spreads the released heat. This faster PCD at Alfvénic speeds could well be responsible for the rapid propagation $\left(400 \mathrm{~km} \mathrm{~s}^{-1}\right)$ we see in our observations. The slope of the PCD with modest speed can also be affected by the heating from the currents (g). The animation accompanying Figure 5 shows how the currents that are created during the spicule formation propagate rapidly into the corona (at Alfvénic speeds) and appear to "meander" through the coronal volume, similar to what is seen in observations of coronal loop strands that often appear to "move" perpendicularly to their own axis. In our simulation, the spicules are thus the harbingers of significant coronal heating both through heating of spicular plasma and heating from current dissipation associated with the spicule. The current dissipation in the corona of our model occurs because of numerical resistivity (Gudiksen et al. 2011). In the solar atmosphere, the dissipation of this energy could be because of current dissipation on small scales or dissipation of the Alfvén waves that are triggered when the spicule is formed (Martínez-Sykora et al. 2017). 

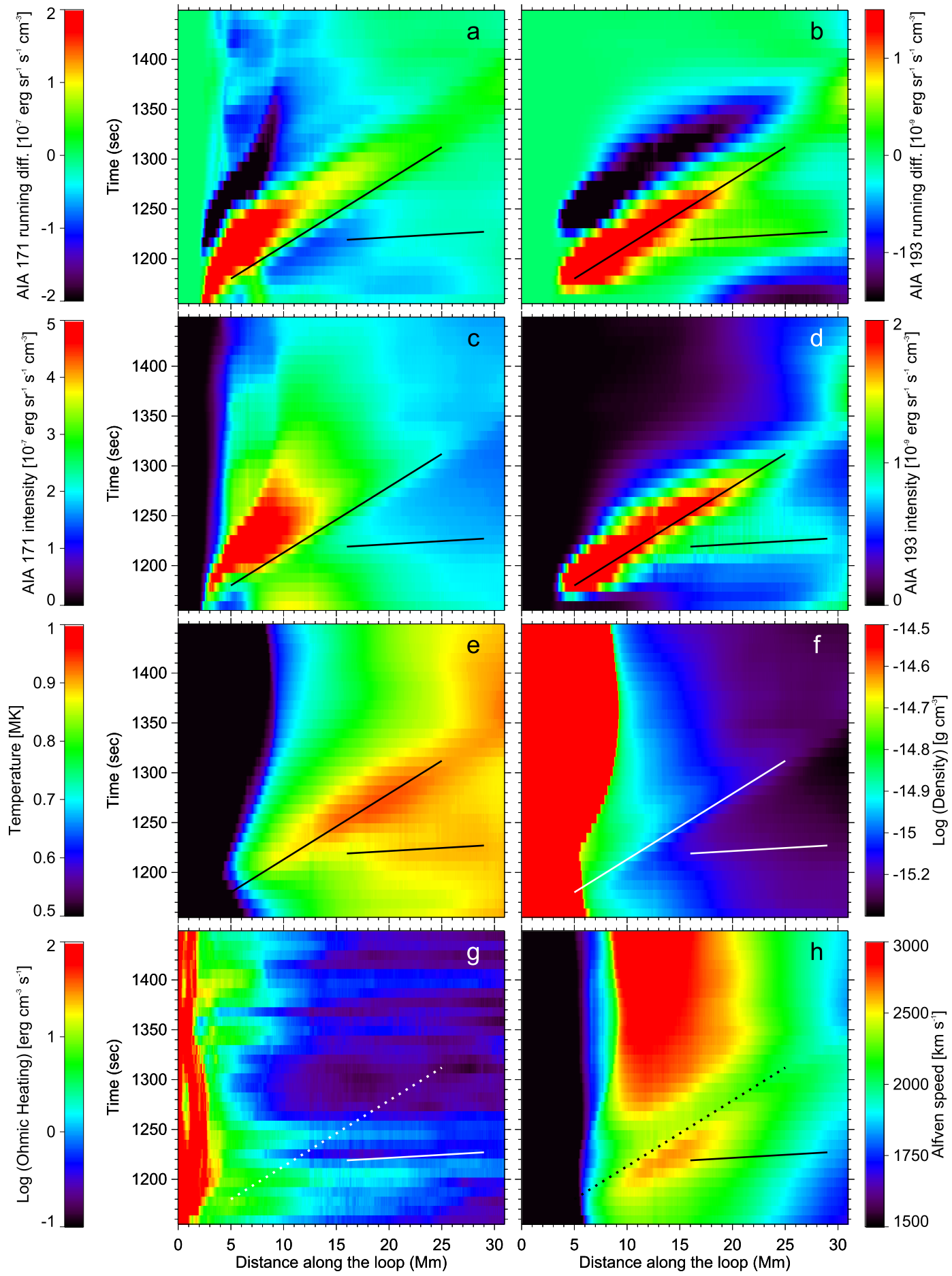

Figure 5. Spacetime plots from 2.5D MHD simulations of type II spicules showing (a) running-difference intensity of Fe IX $171 \AA$, (b) same as for Fe XII $193 \AA$, (c) intensity of Fe IX, (d) same as for Fe XII, (e) temperature, (f) density, (g) Ohmic heating, and (h) Alfvén speed. Black lines show the slopes of the apparent motion of the two different PCDs.

(An animation of this figure is available.)

\section{Discussion}

Our results support a scenario in which "PCDs" along loops originating from plage or strong network regions are not necessarily only a signature of magneto-acoustic waves, but are often caused by a complex sequence of events that involves generation of spicular flows and associated shock waves that propagate into the corona, as well as plasma heating through dissipation of electrical currents and magnetic waves. These currents are a key component of the spicule formation that critically depends on ambipolar diffusion caused by the interaction between ions and neutrals. Our observations provide a detailed view of how spicules, heated from chromospheric to TR temperatures, set off PCDs, but also lead to the formation of new coronal loop strands, thus locally contributing to the mass and energy balance of the corona. Our results suggest that analysis of PCDs through running differencing 
misses the fact that plage-related loop strands are continuously formed and persist after PCDs have "passed." Our results provide a natural explanation for the often confusing reports of apparent speeds, which in our simulations are caused by a mixture of real mass motions of coronal plasma in response to spicular flows and heating, remnants of shock waves generated during the spicule formation, heating through spicule-associated currents, and subsequent thermal conduction. Our simulations also show that idealized "single-field line" approaches (e.g., Klimchuk 2012) to spicule-associated coronal heating are bound to fail: the spicular environment is highly complex, takes place on many neighboring "field lines" some of which carry accelerated plasma that is heated by ambipolar dissipation of electrical currents, and others carry plasma that is heated by spicule-associated currents that rapidly propagate into the corona.

The complexity of physical mechanisms in these simulations highlights why it is so difficult to determine the "spicule contribution to coronal heating." Such an endeavor is driven by an approach that is based on observational phenomena ("chromospheric" spicules) which our simulated (and likely solar) reality defies: spicules are neither chromospheric, TR, or coronal phenomena; they are all of the above, and their dynamics and energetics are intimately tied to that of the corona. Our results indicate that the currents and waves associated with spicule formation should not be ignored in future studies of coronal heating.

IRIS is a NASA small explorer developed and operated by LMSAL with major contributions to downlink communications by ESA and Norwegian Space Centre. This work is supported by NASA (NNG09FA40C; IRIS) and the UK Science and Technology Facilities Council and EU Horizon 2020 research programme (grant No. 647214). The simulations were run on Pleiades (project s1061).

\section{ORCID iDs}

B. De Pontieu (10) https://orcid.org/0000-0002-8370-952X

I. De Moortel (iD https://orcid.org/0000-0002-1452-9330

J. Martinez-Sykora (i) https://orcid.org/0000-0002-0333-5717

S. W. McIntosh (ib https://orcid.org/0000-0002-7369-1776

\section{References}

Athay, R. G., \& Holzer, T. E. 1982, ApJ, 255, 743

Beckers, J. M. 1968, SoPh, 3, 367
Bryans, P., McIntosh, S. W., De Moortel, I., \& De Pontieu, B. 2016, ApJL, 829, L18

Culhane, J. L., Harra, L. K., James, A. M., et al. 2007, SoPh, 243, 19

De Moortel, I., Antolin, P., \& Van Doorsselaere, T. 2015, SoPh, 290, 399

de Moortel, I., Hood, A. W., Ireland, J., \& Walsh, R. W. 2002a, SoPh, 209, 89 de Moortel, I., Ireland, J., Walsh, R. W., \& Hood, A. W. 2002b, SoPh, 209, 61

De Moortel, I., \& Nakariakov, V. M. 2012, RSPTA, 370, 3193

De Moortel, I. 2009, SSRv, 149, 65

De Pontieu, B., Erdélyi, R., \& De Moortel, I. 2005, ApJL, 624, L61

De Pontieu, B., McIntosh, S., Hansteen, V. H., et al. 2007, PASJ, 59, 655

De Pontieu, B., \& McIntosh, S. W. 2010, ApJ, 722, 1013

De Pontieu, B., McIntosh, S. W., Carlsson, M., et al. 2011, Sci, 331, 55

De Pontieu, B., McIntosh, S. W., Hansteen, V. H., \& Schrijver, C. J. 2009, ApJL, 701, L1

De Pontieu, B., Title, A. M., Lemen, J. R., et al. 2014, SoPh, 289, 2733

Gudiksen, B. V., Carlsson, M., Hansteen, V. H., et al. 2011, A\&A, 531, A154

Hansteen, V. H., De Pontieu, B., Rouppe van der Voort, L., van Noort, M., \& Carlsson, M. 2006, ApJL, 647, L73

Hara, H., Watanabe, T., Harra, L. K., et al. 2008, ApJL, 678, L67

Henriques, V. M. J., Kuridze, D., Mathioudakis, M., \& Keenan, F. P. 2016, ApJ, 820, 124

Klimchuk, J. A. 2012, JGRA, 117, A12102

Kosugi, T., Matsuzaki, K., Sakao, T., et al. 2007, SoPh, 243, 3

Lemen, J. R., Title, A. M., Akin, D. J., et al. 2012, SoPh, 275, 17

Madjarska, M. S., Vanninathan, K., \& Doyle, J. G. 2011, A\&A, 532, L1

Martínez-Sykora, J., De Pontieu, B., Carlsson, M., \& Hansteen, V. 2016, ApJL, 831, L1

Martínez-Sykora, J., De Pontieu, B., \& Hansteen, V. 2012, ApJ, 753, 161

Martínez-Sykora, J., De Pontieu, B., Hansteen, V., et al. 2017, Sci, 356, 1269

Martínez-Sykora, J., De Pontieu, B., Leenaarts, J., et al. 2013, ApJ, 771, 66

Martínez-Sykora, J., Hansteen, V., \& Moreno-Insertis, F. 2011, ApJ, 736, 9

McIntosh, S. W., \& De Pontieu, B. 2009, ApJL, 706, L80

Ofman, L., Wang, T. J., \& Davila, J. M. 2012, ApJ, 754, 111

Olluri, K., Gudiksen, B. V., Hansteen, V. H., \& De Pontieu, B. 2015, ApJ, 802,5

Patsourakos, S., Klimchuk, J. A., \& Young, P. R. 2014, ApJ, 781, 58

Pereira, T. M. D., De Pontieu, B., \& Carlsson, M. 2012, ApJ, 759, 18

Pereira, T. M. D., De Pontieu, B., Carlsson, M., et al. 2014, ApJL, 792, L15

Petralia, A., Reale, F., Orlando, S., \& Klimchuk, J. A. 2014, A\&A, 567, A70

Pneuman, G. W., \& Kopp, R. A. 1978, SoPh, 57, 49

Rathore, B., Carlsson, M., Leenaarts, J., \& De Pontieu, B. 2015, ApJ, 811, 81

Rouppe van der Voort, L., De Pontieu, B., Pereira, T. M. D., Carlsson, M., \& Hansteen, V. 2015, ApJL, 799, L3

Rouppe van der Voort, L., Leenaarts, J., de Pontieu, B., Carlsson, M., \& Vissers, G. 2009, ApJ, 705, 272

Samanta, T., Pant, V., \& Banerjee, D. 2015, ApJL, 815, L16

Skogsrud, H., Rouppe van der Voort, L., De Pontieu, B., \& Pereira, T. M. D. 2015, ApJ, 806, 170

Sterling, A. C. 2000, SoPh, 196, 79

Tian, H., McIntosh, S. W., Wang, T., et al. 2012, ApJ, 759, 144

Tripathi, D., \& Klimchuk, J. A. 2013, ApJ, 779, 1

Tsiropoula, G., Tziotziou, K., Kontogiannis, I., et al. 2012, SSRv, 169, 181

Tsuneta, S., Ichimoto, K., Katsukawa, Y., et al. 2008, SoPh, 249, 167

Verwichte, E., Marsh, M., Foullon, C., et al. 2010, ApJL, 724, L194

Wang, T., Ofman, L., \& Davila, J. M. 2013, ApJL, 775, L23

Wilhelm, K., Curdt, W., Marsch, E., et al. 1995, SoPh, 162, 189 